International Journal of Pure and Applied Mathematics

Volume 87 No. 4 2013, 645-656

ISSN: 1311-8080 (printed version); ISSN: 1314-3395 (on-line version)

url: http://www.ijpam.eu

doi: http://dx.doi.org/10.12732/ijpam.v87i4.13

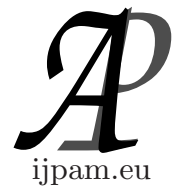

\title{
KOROVKIN SETS FOR SEQUENCES OF SHAPE-PRESERVING OPERATORS
}

\author{
Sergei Sidorov \\ Department of Mechanics and Mathematics \\ Saratov State University \\ Saratov, RUSSIAN FEDERATION
}

\begin{abstract}
This paper examines Korovkin sets for sequences of operators which are shape-preserving relative to a cone of generalized convex functions, and gives some applications to shape-preserving linear approximation.
\end{abstract}

AMS Subject Classification: 41A35, 41A29

Key Words: Korovkin-type theorem, shape-preserving approximation

\section{Introduction}

Let $\mathbf{L}$ be a class of bounded operators on a Banach space $E$ equipped with the norm $\|\cdot\|_{E}$. Let $I$ be the identity operator, i.e. identity embedding operator of $E$ onto $E$.

A finite-dimensional subspace $S$ of $E$ is said to be $\mathbf{L}$-Korovkin set for operator $I$ in $E$, if for any sequence of operators $\left\{L_{n}\right\}$ in $\mathbf{L}$ the convergence of $L_{n} f$ to $I f$ for all $f \in S$ (in the norm $\|\cdot\|_{E}$ ) implies the convergence of $L_{n} f$ to $I f$ for all $f \in E$ (in the norm $\|\cdot\|_{E}$ ).

The case when $\mathbf{L}$ is the set of linear positive operators is deeply examined in [1], [2], [3], [4], [5], [6], [7], [8], [9].

In various applications it is necessary to approximate a function preserving such its shape properties as monotonicity, convexity, concavity, etc. In the the-

Received: July 25, 2013

(c) 2013 Academic Publications, Ltd. url: www.acadpubl.eu 
ory of shape preserving approximation by means of polynomials and splines the last 25 years have seen extensive research. The most significant results were summarized in [10], [11]. Due to increased attention to shape-preserving approximation, properties of shape-preserving operators are of interest. Korovkintype theorems for sequences of operators preserving shape properties of approximated functions relative to signs of fixed orders derivatives was presented in [16].

Using ideas and methods of [2], [3], [4], [5], [9], this paper characterizes Korovkin sets for the identity operator $I$ for the set of linear operators which are shape-preserving relative to a cone of generalized convex functions.

\section{The Cone of Generalized Convex Functions}

Let $C^{k}[0,1]$ be the space of all real-valued and $k$-times continuously differentiable functions defined on $[0,1]$. Let $B^{k}[0,1]$ denote the space of all functions whose derivatives of order $k$ are bounded on $[0,1]$.

A function $f$, defined on $[0,1]$, is said to be convex relative to the system $\left\{u_{0}, \ldots, u_{p}\right\}$ (we will write $f \in C\left(u_{0}, \ldots, u_{p}\right)$ ), if

$$
\left|\begin{array}{cccc}
u_{0}\left(t_{0}\right) & u_{0}\left(t_{1}\right) & \ldots & u_{0}\left(t_{p+1}\right) \\
\ldots & \ldots & \ldots & \ldots \\
u_{p}\left(t_{0}\right) & u_{p}\left(t_{1}\right) & \ldots & u_{p}\left(t_{p+1}\right) \\
f\left(t_{0}\right) & f\left(t_{1}\right) & \ldots & f\left(t_{p+1}\right)
\end{array}\right| \geq 0
$$

for all choices of $0<t_{0}<t_{1}<\ldots<t_{p+1}<1$.

In particular, if $u_{0} \equiv 1$, then $C\left(u_{0}\right)$ is a cone of all increasing functions on $(0,1)$. If $u_{0} \equiv 1, u_{1}(x)=x$, then $C\left(u_{0}, u_{1}\right)$ is a cone of all convex functions on $(0,1)$. The review of some results of the theory of generalized convex functions can be found in the book [12].

Let $k$ be an integer, $\sigma=\left(\sigma_{0}, \ldots, \sigma_{k}\right) \in R^{k+1}, \sigma_{p} \in\{-1,0,1\}$ and $\sigma_{0} \sigma_{k} \neq 0$.

We will suppose that functions $\left\{u_{0}, \ldots, u_{k-1}\right\}$ are linearly independent on $[0,1]$.

Denote $V_{p+1}:=\left\{f \in C^{p+1}[0,1]: f \in C\left(u_{0}, \ldots, u_{p}\right)\right\}, p=0, \ldots, k-1$, $V_{0}:=\{f \in C[0,1]: f \geq 0\}$. Following ideas of [16] let us consider the cone

$$
V_{0, k}(\sigma)=\cap_{p=0}^{k} \sigma_{p} V_{p}
$$

A linear operator $L: C^{k}[0,1] \rightarrow B^{k}[0,1]$ is said to be conservative relative to the cone $V_{0, k}(\sigma)$, if

$$
L\left(V_{0, k}(\sigma)\right) \subset V_{0, k}(\sigma)
$$


Note that a linear operator satisfying (2) is not necessary positive. Let $\mathbf{L}_{0, k}(\sigma)$ denote the set of all linear operators defined in $C^{k}[0,1]$, with range in $B^{k}[0,1]$, satisfying the shape-preserving property

$$
L\left(V_{0, k}(\sigma)\right) \subset \sigma_{0} V_{0} .
$$

The main goal of this paper is to characterize $\mathbf{L}_{0, k}(\sigma)$-Korovkin sets for identity operator $I$ in $C^{k}[0,1]$. Since the set of operators satisfying (2) is subset of the set of operators satisfying (3), all sufficient results which are valid for the set of operators $\mathbf{L}_{0, k}(\sigma)$, will also be valid for the set of all operators which are conservative relative to the cone $V_{0, k}(\sigma)$. Due to linearity of the operators we will assume that $\sigma_{0}=1$.

Following ideas of [13], Bernstein-type operators that are conservative relative to some cone of generalized convex function were examined in [14].

\section{The Characterization of Korovkin Sets}

For a bounded linear operator $L$, let $L^{*}$ denote the adjoint of $L$. Given a point $x \in[0,1]$, let $\delta_{x} \in E^{*}$ denote Dirac functional, i.e. point evaluation of $f$ at $x$, $\delta_{x}(f)=f(x), f \in E$.

Let $F$ be a subset of $E^{*}$ and let $\mathbf{L}$ be the set of all bounded linear operators $L$ on $E$, such that $L^{*} \delta_{x} \in F$ for all $x \in[0,1]$. For example, if $F$ is the set of all positive functionals on $E$, then $\mathbf{L}$ would be the set of all positive operators.

Let $\lambda \in F$ be a functional and let $S$ be a finite-dimensional subspace of $E$.

The set $S$ is said to be $F$-Korovkin set for $\lambda$, if for any sequence $\left\{\lambda_{n}\right\} \subset F$ the convergence of $\lambda_{n}(f) \rightarrow \lambda(f)$ for all $f \in S$ implies the convergence $\lambda_{n}(f) \rightarrow$ $\lambda(f)$ for all $f \in E$.

Following [5], $S$ is said to be $F$-determining set for $\lambda$, if for any functional $\mu \in F$ the equality $\mu(f)=\lambda(f)$ for all $f \in S$ implies $\mu=\lambda$.

Given $\mu \in E^{*}$ and $A \subset E$, let $\left.\mu\right|_{A} \in A^{*}$ denotes the functional $\mu$ restricted to $A$.

This section presents necessary and sufficient conditions of $\mathbf{L}_{0, k}(\sigma)$-Korovkin sets for identity operator $I$ in $E=C^{k}[0,1]$.

Let $V_{0, k}^{*}(\sigma)$ denote the set of all linear functionals in $E^{*}$ which are nonnegative on $V_{0, k}(\sigma)$.

Given $\mathbf{y}=\left(y_{1}, \ldots, y_{r}\right) \in[0,1]^{r}, r \in \mathbb{N}, 0 \leq y_{1}<\ldots<y_{r} \leq 1$, let us denote $\delta_{\mathbf{y}}=\left(\delta_{y_{1}}, \ldots, \delta_{y_{r}}\right)$.

Given $\alpha \in \mathbb{R}^{r}$ and $\mathbf{y}=\left(y_{1}, \ldots, y_{r}\right) \in[0,1]^{r}$, denote $\alpha \delta_{\mathbf{y}}=\sum_{i=1}^{r} \alpha_{i} \delta_{y_{i}} \in E^{*}$. The expression $\left.\alpha \delta_{\mathbf{y}}\right|_{V_{0, k}(\sigma)} \geq 0$ means that for any $f \in V_{0, k}(\sigma)$ the inequality 
$\alpha \delta_{\mathbf{y}}(f) \geq 0$ holds. Let us denote

$$
V^{*}(\mathbf{y}):=\left\{\alpha \in \mathbb{R}^{r}:\left.\alpha \delta_{\mathbf{y}}\right|_{V_{0, k}(\sigma)} \geq 0\right\}
$$

Theorem 1. The following are equivalent:

1. for any $x \in[0,1]$ the finite-dimensional subspace $S$ is $V_{0, k}^{*}(\sigma)$-determining set for $\delta_{x}$.

2. $\forall r \in \mathbb{N}, \forall \mathbf{y}=\left(y_{1}, \ldots, y_{r}\right) \in[0,1]^{r}, \forall x \in[0,1], x \neq y_{i}, \nexists \alpha \in V^{*}(\mathbf{y})$, such that $\left.\delta_{x}\right|_{S}=\left.\alpha \delta_{\mathbf{y}}\right|_{S}$.

Proof. Assume that the proposition 1 of Theorem holds. Let $r \in \mathbb{N}, x \in$ $[0,1], \mathbf{y} \in[0,1]^{r}, \alpha \in V^{*}(\mathbf{y})$ be such that

$$
\left.\delta_{x}\right|_{S}=\left.\alpha \delta_{\mathbf{y}}\right|_{S}
$$

Then values of functionals $\delta_{x}, \delta_{x} \in V_{0, k}^{*}(\sigma)$, and $\lambda=\alpha \delta_{\mathbf{y}}, \lambda \in V_{0, k}^{*}(\sigma)$, coincide on every function of the set $S$ and differ on every function $g \in E$, such that $\delta_{x}(g)=1, \delta_{y_{i}}(g)=0, i=1, \ldots, r$.

Assume that the proposition 2 of Theorem holds. Suppose that for a point $x \in[0,1]$ there exists a functional $\mu \in V_{0, k}^{*}(\sigma), \mu \neq \delta_{x}$, such that $\left.\mu\right|_{S}=\left.\delta_{x}\right|_{S}$.

Consider the set

$$
M=\left\{\left.\alpha \delta_{\mathbf{y}}\right|_{S}: \mathbf{y} \in[0,1]^{r}, \alpha \in V^{*}(\mathbf{y}), r \in \mathbb{N}\right\}
$$

Let $m$ denote the dimension of the set $S, m=\operatorname{dim} S$. The set $M$ is a convex subset of $\mathbb{R}^{m}$. It follows from Carateodory's theorem that there exist $\beta_{i} \geq 0$, $\mathbf{y}^{i} \in[0,1]^{r_{i}}, \alpha_{i} \in V^{*}\left(\mathbf{y}^{i}\right), i=1, \ldots, m+1, r_{i} \in N$, such that

$$
\mu(f)=\sum_{i=1}^{m+1} \beta_{i} \alpha_{i} \delta_{\mathbf{y}^{i}}(f)
$$

for every $f \in S$. This contradicts the proposition 2 of Theorem.

Lemma 2. If $\forall x \in[0,1]$ a finite-dimensional set $S$ is $V_{0, k}^{*}(\sigma)$-determining set for $\delta_{x}$, then the set $S$ is $V_{0, k}^{*}(\sigma)$-Korovkin set for $\delta_{x}$ for every $x \in[0,1]$.

Proof. It is necessary to show that if a sequence $\left\{\lambda_{n}\right\} \subset V_{0, k}^{*}(\sigma)$ be such that

$$
\lambda_{n}(f) \rightarrow \delta_{x}(f)
$$

for every $f \in S$, then $\lambda_{n}(f) \rightarrow \delta_{x}(f)$ for every $f \in E$. 
First it should be shown that $\left\{\left\|\lambda_{n}\right\|\right\}$ is bounded above.

Let us denote

$$
M=\left\{\alpha \delta_{\mathbf{y}}: \mathbf{y} \in[0,1]^{r}, \alpha \in V^{*}(\mathbf{y}), r \in \mathbb{N}\right\} .
$$

The set $M \subset \mathbb{R}^{m}$ is convex, $m=\operatorname{dim} S$, and does not contain the origin of the space $R^{m}, 0_{m} \notin M$. Indeed, if $0_{m} \in M$ then there exist $\mathbf{y} \in[0,1]^{r}$ and $\alpha \in V^{*}(\mathbf{y})$, such that

$$
\left.\alpha \delta_{\mathbf{y}}\right|_{S}=0 .
$$

This contradicts the proposition 2 of Theorem 1. It follows from Separation Theorem that in the space $\mathbb{R}^{m}$ there exists hyperplane separating $0_{m}$ and $M$. Therefore, there are $f \in S$ and $d>0$, such that the inequality $\alpha \delta_{\mathbf{y}}>d$ holds for every $\mathbf{y} \in[0,1]^{r}$ and $\alpha \in V^{*}(\mathbf{y})$.

It follows from (5) that $\lim _{n \rightarrow \infty} \lambda_{n}(f)=\delta_{x}(f)$, and consequently, $\lambda_{n}(f)<$ $M<\infty$. I.e., for any $g$ such that $|g|=1, \alpha \delta_{\mathbf{y}}(g)=d$, we have $\lambda_{n}(g)<M$, and therefore $\left\|\lambda_{n}\right\|<\frac{M}{d}$.

Since $\left\{\lambda_{n}\right\}$ satisfies (5), for any $\varepsilon>0$ there exist such $\mathbf{y} \in[0,1]^{r}, \alpha \in V^{*}(\mathbf{y})$, that

$$
\left|\lambda_{n}(f)-\alpha \delta_{\mathbf{y}}(f)\right|<\varepsilon .
$$

Since $E$ is a separable space, any bounded sequence $\lambda_{n} \in E^{*}$ is a weakly compact sequence. Therefore, there exists a weakly convergent subsequence of $\lambda_{n} \in E^{*}$. Moreover, the sequence $\lambda_{n}$ will be converge weakly to functional $\delta_{x}$, since each accumulation (limit) functional $\lambda$ of the sequence must satisfy equalities

$$
\left.\lambda\right|_{S}=\left.\delta_{x}\right|_{S}
$$

and therefore, coincide with $\delta_{x}$.

Theorem 3. A finite-dimensional subspace $S$ is $\mathbf{L}_{0, k}(\sigma)$-Korovkin set for the identity operator $I$ in $E=C^{k}[0,1]$, if and only if for each $x \in[0,1]$ the set $S$ is $V_{0, k}^{*}(\sigma)$-determining set for $\delta_{x}$.

Proof. First it should be proved necessary condition of Theorem. Assume that $S$ is Korovkin set. Suppose that for a point $x_{0} \in[0,1]$ there exists two different linear functionals $\mu, \lambda \in V_{0, k}^{*}(\sigma)$, such that $\left.\mu\right|_{S}=\left.\lambda\right|_{S}=\delta_{x_{0}}$. For simplicity we will suppose $\left.\lambda\right|_{S}=\delta_{x_{0}}$. Then, as it was shown in the proof of Theorem 1, there exist $r \in \mathbb{N}, \mathbf{y} \in[0,1]^{r}$ and $\alpha \in V^{*}(\mathbf{y})$, such that

$$
\left.\delta_{x_{0}}\right|_{S}=\left.\alpha \delta_{\mathbf{y}}\right|_{S} .
$$


Define a linear operator $L_{0}: C^{k}[0,1] \rightarrow B^{k}[0,1]$ by

$$
L_{0} f(x)=\left\{\begin{array}{l}
f(x), x \neq x_{0} \\
\alpha \delta_{\mathbf{y}}(f), x=x_{0}
\end{array}\right.
$$

Since $\alpha \in V^{*}(\mathbf{y})$, we have $L_{0}\left(V_{0, k}(\sigma)\right) \subset \sigma_{0} V_{0}$. It follows from equalities (6) that $L_{0}$ is the identity operator on the subspace $S$, i.e. $L_{0} f=I f=f$ for all $f \in S$.

Let

$$
L_{n} f=L_{0} f, n=1,2, \ldots .
$$

Then the sequence $\left\{L_{n}\right\}_{n \geq 0}$ be such that

1. $L_{n}\left(V_{0, k}(\sigma)\right) \subset \sigma_{0} V_{0}$;

2. $\lim _{n \rightarrow \infty}\left\|\left(L_{n}-I\right) f\right\|=0$ for all $f \in S$.

On the other hand, for every $f \in E$ such that $\delta_{x_{0}}(f)=1$ and $\delta_{\mathbf{y}}(f)=0_{r} \in$ $\mathbb{R}^{r}$, we have $L_{0} f \neq f$, i.e. $S$ is not $\mathbf{L}_{0, k}(\sigma)$-Korovkin set for $I$ in $E$.

To prove the sufficient condition of Theorem, we assume that $S$ is $V_{0, k}^{*}(\sigma)$ Korovkin set for $\delta_{x}$ for all $x \in[0,1]$. Let $\left\{L_{n}\right\}_{n \geq 1}, L_{n}: C^{k}[0,1] \rightarrow B^{k}[0,1]$, be a sequence of such linear operators, that

1. $L_{n}\left(V_{0, k}(\sigma)\right) \subset \sigma_{0} V_{0}$;

2. for every $f \in S$

$$
\lim _{n \rightarrow \infty}\left\|\left(L_{n}-I\right) f\right\|=0 .
$$

It is well-known that a sequence $\left\{f_{n}\right\} \subset E$ converges uniformly to $f \in E$ if and only if for each $x \in[0,1] f_{n}\left(x_{n}\right) \rightarrow f(x)$ as $n \rightarrow \infty$ for every sequence $\left\{x_{n}\right\} \subset[0,1]$ such that $x_{n} \rightarrow x$ as $n \rightarrow \infty$.

Let $\left\{x_{n}\right\} \subset[0,1]$ be a sequence such that $x_{n} \rightarrow x$. For every $f \in S$ we have $L_{n} f\left(x_{n}\right) \rightarrow f(x)$, or, in the other words, $L_{n}^{*} \delta_{x_{n}}(f) \rightarrow \delta_{x}(f)$. It follows from Lemma 2 that $L_{n}^{*} \delta_{x_{n}}(f) \rightarrow \delta_{x}(f)$ for every $f \in E$, i.e. $L_{n} f \rightarrow f$ for every $f \in E$, and therefore, $S$ is Korovkin set for the identity operator $I$. 


\section{Corollaries and Applications}

Throughout this section it is assumed that the system of functions $\left\{u_{0}, \ldots, u_{k-1}\right\}$, $u_{l} \in C^{k-1}[0,1], l=0, \ldots, k-1$, is an extended complete Tchebychev (ECT) system on $[0,1]$. The cone $V_{0, k}(\sigma)$ is defined in (1) and based on the system $\left\{u_{0}, \ldots, u_{k-1}\right\}$.

First it should be proved two preliminary lemmas.

Let $L_{k-1} f\left(\cdot ; y_{0}, y_{1}, \ldots, y_{k-1}\right) \in \operatorname{span}\left\{u_{0}, \ldots, u_{k-1}\right\}$ denotes the generalized polynomial, which interpolates $f \in E$ at points $0 \leq y_{0}<y_{1}<\ldots<y_{k-1}<1$ :

$$
L_{k-1} f\left(y_{i} ; y_{0}, y_{1}, \ldots, y_{k-1}\right)=f\left(y_{i}\right), i=0, \ldots, k-1 .
$$

Let us set $y_{-1}=-\infty, y_{k}=+\infty$.

Lemma 4. Let $f \in V_{0, k}(\sigma)$.

1. If $\sigma_{0} \sigma_{k}>0$, then for all $x \in \bigcup_{i=0}^{[(k-1) / 2]}\left[y_{k-1-(2 i+1)}, y_{k-1-2 i}\right]$

$$
\sigma_{0} L_{k-1} f\left(x ; y_{0}, \ldots, y_{k-1}\right) \geqslant 0 .
$$

2. If $\sigma_{0} \sigma_{k}<0$, then for all $x \in \bigcup_{i=-1}^{[(k-2) / 2]}\left[y_{k-1-(2 i+2)}, y_{k-1-(2 i+1)}\right]$ the inequality (9) holds.

Proof. Suppose that $x \in\left(y_{l-1}, y_{l}\right), l=0, \ldots, k$. It follows from $f \in V_{0, k}(\sigma)$, that

$$
\sigma_{k} \Delta_{k-1} f\left(x ; y_{0}, \ldots, y_{k-1}\right) \geqslant 0
$$

where

$$
\Delta_{k-1} f\left(x ; y_{0}, \ldots, y_{k-1}\right)=(-1)^{l}\left|\begin{array}{cccc}
u_{0}(x) & u_{0}\left(y_{0}\right) & \ldots & u_{0}\left(y_{k-1}\right) \\
\ldots & \ldots & \ldots & \ldots \\
u_{k-1}(x) & u_{k-1}\left(y_{0}\right) & \ldots & u_{k-1}\left(y_{k-1}\right) \\
f(x) & f\left(y_{0}\right) & \ldots & f\left(y_{k-1}\right)
\end{array}\right| .
$$

It follows from

$$
\begin{aligned}
& \Delta_{k-1} f\left(x ; y_{0}, \ldots, y_{k-1}\right)= \\
& \quad=(-1)^{k-1+l}\left(L_{k-1} f\left(x ; y_{0}, \ldots, y_{k-1}\right)-f(x)\right) \operatorname{det}\left(u_{i}\left(y_{j}\right)\right)_{i=0, \ldots, k-1}^{j=0, \ldots, k-1},
\end{aligned}
$$

that $\sigma_{k}(-1)^{k-1+l} L_{k-1} f\left(x ; y_{0}, \ldots, y_{k-1}\right) \geqslant \sigma_{k}(-1)^{k-1+l} f(x)$. Since $\sigma_{0} f \geqslant 0$, the inequality (9) holds for appropriate $x$.

We need the following property of interpolatory polynomials. 
Lemma 5. Let $L_{k-1} f\left(\cdot ; y_{0}, y_{1}, \ldots, y_{k-1}\right) \in \operatorname{span}\left\{u_{0}, \ldots, u_{k-1}\right\}$ be the polynomial, which interpolates $f$ at points $0 \leq y_{0}<y_{1}<\ldots<y_{k-1}<1$. Then

$$
L_{k-1} u_{i}\left(\cdot ; y_{0}, \ldots, y_{k-1}\right)=u_{i}, \quad i=0, \ldots, k-1 \text {. }
$$

The following three propositions are main results of this section.

Corollary 6. Assume that $S$ is $\boldsymbol{L}_{0, k}(\sigma)$-Korovkin set for the identity operator $I$ in $C^{k}[0,1]$. If $\left\{u_{0}, \ldots, u_{k-1}\right\} \subset S$, then $\operatorname{dim} S \geq k+1$.

Proof. It should be shown that if $\operatorname{dim} S=k$, then there exists a sequence of linear operators $\left\{L_{n}\right\} \in \mathbf{L}_{0, k}(\sigma)$, such that

1. $L_{n} f=f$ for all $f \in S$;

2. there exists such $g \in E$ and $\theta \in(0,1)$, that $\lim _{n \rightarrow \infty} L_{n} g(\theta) \neq g(\theta)$.

Take arbitrary points $0<y_{1}<y_{2}<\ldots<y_{k}<1$. Let us denote by

$$
L_{k-1} f\left(\cdot ; y_{0}, y_{1}, \ldots, y_{k-1}\right) \in \operatorname{span}\left\{u_{0}, \ldots, u_{k-1}\right\}
$$

the generalized polynomials which interpolates $f \in E$ at points $0<y_{0}<y_{1}<$ $\ldots<y_{k-1}<1$.

It follows from Lemma 4 that there exists a point $\theta \in(0,1), \theta \neq y_{i}, i=$ $1, \ldots, k$, such that

$$
L_{k-1} f\left(\theta ; y_{0}, y_{1}, \ldots, y_{k-1}\right) \geq 0
$$

for all $f \in V_{0, k}(\sigma)$

Given $n \in N$, let us define

$$
L_{n} f(x)=\left\{\begin{array}{l}
f(x), x \neq \theta \\
L_{k-1} f\left(\theta ; y_{0}, y_{1}, \ldots, y_{k-1}\right) .
\end{array}\right.
$$

It is obvious that operators $L_{n} \in \mathbf{L}_{0, k}(\sigma)$, i.e. $L_{n}\left(V_{0, k}(\sigma)\right) \subset V_{0}$. It follows from Lemma 5 that $L_{n} f=f$ on $[0,1]$ for all $f \in S$. On the other hand, let a function $g \in E$ be such that

$$
\operatorname{det}\left(u_{i}\left(y_{j}\right)\right)_{i=0, \ldots, k}^{j=0, \ldots, k} \neq 0,
$$

where $y_{0}:=\theta, u_{k}:=g$. It follows from (10) that

$$
L_{k-1} g\left(\theta ; y_{0}, y_{1}, \ldots, y_{k-1}\right)-g(\theta) \neq 0 \text {. }
$$

Then we have $\lim _{n \rightarrow \infty} L_{n} g(\theta) \neq g(\theta)$. 
Denote $e_{j}(x)=x^{j}, j=0,1, \ldots$

Corollary 7. Let $k \geq 2$, and $u_{k} \in C^{k}[0,1]$ be such that system $u_{0}, u_{1}, \ldots, u_{k}$ is ECT-system on [0,1]. If $u_{0}=e_{0}, u_{1}=e_{1}$ and $\sigma_{0} \sigma_{2} \neq-1$, then $S=$ $\operatorname{span}\left\{u_{0}, \ldots, u_{k}\right\}$ is $\boldsymbol{L}_{0, k}(\sigma)$-Korovkin set for the identity operators $I$ in $C^{k}[0,1]$.

Proof. Suppose there exist such $\mathbf{y}=\left(y_{1}, \ldots, y_{r}\right), x \neq y_{i}, x \in[0,1]$, and $\alpha \in V^{*}(\mathbf{y})$, that

$$
\left.\delta_{x}\right|_{S}=\left.\alpha \delta_{\mathbf{y}}\right|_{S} .
$$

On the other hand, there exists [15] such a generalized polynomial $f \in S$, that $\delta_{x}(f)=0$ and $\alpha \delta_{\mathbf{y}}(f)>0$. This contradicts to (11).

Remark 8. The following example shows that in the case $\sigma_{0} \sigma_{2}=-1$, the statement of Corollary 7 is not true. Given $x \in[0,1]$, let us define the functional $\mu_{x}$ by

$$
\begin{aligned}
& \mu_{x}(f)=(1 / 2) \delta_{x}\left(e_{2}\right)[0, s, 2 s] f+(1 / 3) \delta_{x}\left(e_{3}\right)([1-2 s, 1-s, 1] f-[0, s, 2 s] f)+ \\
& +\delta_{x}\left(e_{1}\right)\left(\delta_{1}(f)-\delta_{0}(f)-(1 / 3)[1-2 s, 1-s, 1] f-(1 / 6)[0, s, 2 s] f\right)+\delta_{0}(f)
\end{aligned}
$$

where $s=1 / 4$ and $\left[y_{1}, y_{2}, y_{3}\right] f$ denotes the second order divided difference of $f$ at points $y_{1}, y_{2}, y_{3}$. It easy to check that

1. $\mu_{x} e_{0}=e_{0}(x), \mu_{x} e_{1}=e_{1}(x), \mu_{x} e_{2}=e_{2}(x)$, i.e. representation (11) holds for $S=\operatorname{span}\left\{e_{0}, e_{1}, e_{2}\right\}$ and for any $x \neq i / 4, i=0, \ldots, 4$;

2. $\mu_{x} f \geq 0$ for every $f \in V_{0,2}(\sigma)$, where $\sigma=(1,0,-1)$.

Corollary 9. If $S=\operatorname{span}\left\{u_{0}, \ldots, u_{k}\right\}$ is $\boldsymbol{L}_{0, k}(\sigma)$-Korovkin set for the identity operator $I$ in $C^{k}[0,1]$, then $\left\{u_{0}, \ldots, u_{k}\right\}$ is ECT-system on $[0,1]$.

Proof. Assume the contrary. Then there exist

$$
\mathbf{y}^{1}=\left(y_{0}^{1}, \ldots, y_{k}^{1}\right), \mathbf{y}^{2}=\left(y_{0}^{2}, \ldots, y_{k}^{2}\right) \in[0,1]^{k+1},
$$

satisfying

$$
\operatorname{det}\left(u_{i}\left(y_{j}^{1}\right)\right) \cdot \operatorname{det}\left(u_{i}\left(y_{j}^{2}\right)\right)<0 .
$$

Since $F\left(y_{0}, \ldots, y_{k}\right)=\operatorname{det}\left(u_{i}\left(y_{j}\right)\right)$ is continuous on $\mathbb{R}^{k+1}$, there exists such $\mathbf{y}^{0}=$ $\left(y_{0}^{0}, \ldots, y_{k}^{0}\right) \in[0,1]^{k+1}$, that $\operatorname{det}\left(u_{i}\left(y_{j}^{0}\right)\right)=0$.

It follows from Lemma 4 that there is a point $y_{s}^{0} \in\left\{y_{0}^{0}, \ldots, y_{k}^{0}\right\}$, such that

$$
L_{k-1} f\left(y_{s}^{0} ; y_{0}^{0}, \ldots, y_{s-1}^{0}, y_{s+1}^{0}, \ldots, y_{k-1}^{0}\right) \geq 0,
$$


for all $f \in V_{0, k}(\sigma)$.

Consider the functional

$$
\alpha \delta_{\mathbf{y}}(f):=L_{k-1} f\left(y_{s}^{0} ; y_{0}^{0}, \ldots, y_{s-1}^{0}, y_{s+1}^{0}, \ldots, y_{k-1}^{0}\right)
$$

where $\mathbf{y}=\left(y_{0}^{0}, \ldots, y_{s-1}^{0}, y_{s+1}^{0}, \ldots, y_{k-1}^{0}\right)$. It is clear that $\alpha \delta_{\mathbf{y}} \in V^{*}(\mathbf{y})$. It follows from Lemma 5 and the equality (10), that $\alpha \delta_{\mathbf{y}}(f)=f\left(y_{s}\right)$ for all $f \in$ $\operatorname{span}\left\{u_{0}, \ldots, u_{k}\right\}$.

Let $D^{i}$ denote the $i$-th differential operator, $D^{i} f(x)=d^{i} f(x) / d x^{i}$. Let $k \geq 2$ be an integer, $\sigma=\left(\sigma_{0}, \ldots, \sigma_{k}\right) \in R^{k+1}, \sigma_{p} \in\{-1,0,1\}$ and $\sigma_{0} \sigma_{k} \neq 0$. Let $\|f\|=\sup _{x \in[0,1]}|f(x)|$. In the paper of F. J. Muñoz-Delgado, V. RamírezGonzález and D. Cárdenas-Morales [16] the following cone

$$
C_{0, k}(\sigma)=\left\{f \in C^{k}[0,1]: \sigma_{i} D^{i} f \geq 0,0 \leq i \leq k\right\}
$$

was considered. In particular, they proved [16] the next Korovkin-type result for a sequences of linear operators preserving shape.

Theorem 10. Let $C_{0, k}(\sigma)$ be a cone, such that $\sigma_{0} \sigma_{2} \neq-1$. Let $L_{n}$ : $C^{k}[0,1] \rightarrow C^{k}[0,1], n \geq 1$, be a sequence of linear operators. If

1. $L_{n}\left(C_{0, k}(\sigma)\right) \subset \sigma_{0} V_{0}$,

2. $\lim _{n \rightarrow \infty}\left\|L_{n} e_{j}-e_{j}\right\|=0, j=0, \ldots, k$,

Then $\lim _{n \rightarrow \infty}\left\|L_{n} f-f\right\|=0$ for all $f \in C^{k}[0,1]$.

The result of Theorem 10 follows from Corollary 7 with $u_{j}=e_{j}, j=$ $0, \ldots, k$. It arises from the fact that the system $e_{0}, \ldots, e_{k}$ is extended complete Tchebycheff system on $[0,1]$.

The next proposition follows from Corollary 7.

Corollary 11. Let $C_{0, k}(\sigma)$ be a cone, such that $\sigma_{0} \sigma_{2} \neq-1$. Let $g \in$ $C^{k}[0,1]$ be such that the system $e_{0}, \ldots, e_{k-1}, g$ is an extended complete Tchebycheff system on $[0,1]$. Let $L_{n}: C^{k}[0,1] \rightarrow C^{k}[0,1], n \geq 1$, be a sequence of linear operators. If

1. $L_{n}\left(C_{0, k}(\sigma)\right) \subset \sigma_{0} V_{0}$;

2. $\lim _{n \rightarrow \infty}\left\|L_{n} e_{j}-e_{j}\right\|=0, j=0, \ldots, k-1$,

3. $\lim _{n \rightarrow \infty}\left\|L_{n} g-g\right\|=0$, 
then $\lim _{n \rightarrow \infty}\left\|L_{n} f-f\right\|=0$ for all $f \in C^{k}[0,1]$.

Remark 12. The results of this paper can also be derived using the properties of Minkowski duality and ideas of paper [17].

\section{Acknowledgments}

The work was supported by Russian Fund for Basic Research (project 13-0100238).

\section{References}

[1] P.P. Korovkin, On convergence of linear positive operators in the space of continuous functions, Dokl. Akad. Nauk. SSR, 90, No. 6 (1953), 961-964.

[2] P.P. Korovkin, Conditions for the uniqueness of the moment problem and the convergence of sequences of linear operators, Notes of Kalinin State Pedagogical Institute, 26 (1958), 95-102.

[3] V.I. Volkov, On conditions for convergence of sequences of linear positive operators in the space of continuous functions assigned on closed surfaces, Uspeki Mat. Nauk, 15, No. 1 (1960), 181-184.

[4] Y.A. Šaškin, Korovkin systems in spaces of continuous functions, Izv. Akad. Nauk SSSR, Ser, Mat., 26, No. 4 (1962), 495-512.

[5] Y.A. Šaškin, Finitely defined linear operators in spaces of continuous functions, Uspeki Mat. Nauk, 20, No. 6 (1965), 175-180.

[6] G.G. Lorentz, Korovkin sets, in: Regional Conference at the Univ. of Calif. Riverside, June 15-19, 1972.

[7] J. Cavaretta, A.S., Korovkn theorem for finitely defined operators, in: G.G. Lorentz (Ed.), Approximation Theory, Academic Press (1973), 299-305.

[8] C.A. Micchelli, Chebyshev subspaces and convergence of positive linear operators, Proc. Amer. Math. Soc, 40 (1973), 448-452, doi: 10.2307/2039390.

[9] Le Baron, O. Ferguson, Michael D. Rusk, Korovkin sets for an operator on a space of continuous functions, Pacific J. Math., 65, No 2 (1976), 337-345. 
[10] S. G. Gal, Shape-Preserving Approximation by Real and Complex Polynomials, Birkhäuser Boston, USA (2008), doi: 10.1007/978-0-8176-4703-2.

[11] K. A. Kopotun, D. Leviatan, A. Prymak, and I. A. Shevchuk, Uniform and Pointwise Shape Preserving Approximation by Algebraic Polynomials, Surveys in Approximation Theory, 6 (2011), 24-74.

[12] S. Karlin, W. Stadden, Tchebycheff systems: With applications in analysis and statistics, Vol. Pure and Applied Mathematics XV, Interscience Publishers John Wiley \& Sons, New York (1966).

[13] H. Gonska, P. Piţul, I. Raşa, General King-type operators, Results Math. 53 (3-4) (2009), 279-286, doi: 10.1007/s00025-008-0338-9.

[14] D. Cárdenas-Morales, P. Garrancho, I. Raşa, Bernstein-type operators which preserve polynomials, Comput. Math. Appl. 62 (1) (2011), 158-163, doi: 10.1016/j.camwa.2011.04.063.

[15] S. P. Sidorov, Korovkin-type theorem for sequences of operators preserving shape, Positivity, 15, No. 1 (2011), 1-8, doi: 10.1007/s11117-009-0038-z.

[16] F.J. Muñoz-Delgado, V. Ramírez-González, D. Cárdenas-Morales, Qualitative Korovkin-type results on conservative approximation, J. Approx. Theory, 94 (1998), 144-159, doi: 10.1006/jath.1998.3182.

[17] S.S. Kutateladze, A.M. Rubinov, Minkowsi duality and its applications, Russ. Math. Surv., 27, No. 3 (1972), 127-176. 